\title{
Timing of infliximab and adalimumab initiation despite methotrexate in children with chronic non-infectious anterior uveitis
}

\author{
Courtney McCracken ${ }^{1} \cdot$ Steven Yeh $^{1,2} \cdot$ Kirsten Jenkins $^{3} \cdot$ Curtis Travers $^{1} \cdot$ Daneka Stryker $^{1} \cdot$ Steven Tommasello $^{1}$. \\ Kelly A. Rouster-Stevens ${ }^{1,3}$. Scott R. Lambert ${ }^{4}$ - Sampath Prahalad ${ }^{1,3,5}$ - Carolyn Drews-Botsch ${ }^{1,2,6}$. \\ Sheila T. Angeles-Han (10)
}

Received: 5 January 2018 / Revised: 24 July 2018 / Accepted: 5 November 2018 / Published online: 28 November 2018

(c) The Royal College of Ophthalmologists 2018

\begin{abstract}
Aims Methotrexate (MTX) is standard treatment in pediatric chronic anterior uveitis (CAU). Addition of tumor necrosis factor- $\alpha$ inhibitors (TNFi) is often needed. We describe the timing and risk factors for TNFi use in children with CAU on MTX.

Methods In this retrospective study, we reviewed 51 records, and 46 met inclusion criteria. Primary outcome was the addition of TNFi due to active CAU per Standardization of Uveitis Nomenclature criteria. Time to TNFi and factors associated with their addition were assessed using survival analysis models.

Results Of 46 children treated with MTX for uveitis (36 juvenile idiopathic arthritis-associated uveitis, 10 idiopathic CAU), $72 \%$ had ocular complications. MTX was started a median of 5.0 months, and TNFi 43 months from uveitis diagnosis. Kaplan-Meier estimates suggest that cumulatively, 12\% (95\% CI: 4-23\%) start TNFi within 6 months of MTX, 21\% (12-37\%) within 1 year, and 39\% (24-54\%) within 2 years. On Cox Proportional Hazard regression analysis, children with idiopathic CAU required TNFi earlier in their uveitis course (at 3 months (Hazard Ratio 6.06; 95\% confidence interval (1.25-29.41))). Females appeared less likely to require TNFi early. Children treated in 2012 and later were more likely to receive TNFi earlier than those treated before 2012.

Conclusion Little is known about optimal time to initiate treatment or factors associated with the need to add TNFi in children on MTX. Children with idiopathic CAU and males required TNFi earlier in their course. Factors associated with these potential risk factors for TNFi warrant further investigation.
\end{abstract}

Sheila T. Angeles-Han

Sheila.Angeles-Han@cchmc.org

1 Department of Pediatrics, Emory University School of Medicine, Atlanta, GA, USA

2 Department of Ophthalmology, Emory University School of Medicine, Atlanta, GA, USA

3 Children's Healthcare of Atlanta, Atlanta, GA, USA

4 Department of Ophthalmology, Stanford University, Stanford, GA, USA

5 Department of Genetics, Emory University School of Medicine, Atlanta, GA, USA

6 Rollins School of Public Health, Emory University, Atlanta, GA, USA

7 Division of Rheumatology, Cincinnati Children's Hospital Medical Center and Department of Pediatrics, University of Cincinnati, Cincinnati, OH, USA

\section{Introduction}

Persistent ocular inflammation in children with chronic anterior uveitis (CAU) can lead to severe ocular complications and permanent vision loss [1,2]. Anterior uveitis is the most common location. Pediatric uveitis can occur in isolation, as in idiopathic CAU (iCAU), but it is also commonly associated with juvenile idiopathic arthritis, as in JIA-associated uveitis (JIA-U) [3]. Approximately 50\% of affected children develop ocular complications such as synechiae, glaucoma, and cataracts, 25-40\% experience vision loss, and 10-20\% legal blindness [4-8]. Children with iCAU can present with worse ocular sequelae, as uveitis is usually diagnosed on presentation, as compared with children with JIA who undergo regular ophthalmology screening. Appropriate and timely treatment may improve visual outcomes.

Randomized controlled trials in pediatric uveitis are scarce. Treatment is guided by expert opinion, retrospective 
studies, and panel guidelines [9-11]. Topical glucocorticoids are standard first-line treatment, but in those refractory to glucocorticoids, methotrexate (MTX) is the most commonly prescribed immunosuppressant [12-15]. Emerging data suggest the benefit of use of tumor necrosis factor- $\alpha$ inhibitors (TNFi) in pediatric aged patients [16]. Approximately $73 \%$ of children respond to MTX, but many fail MTX and require TNFi [17-27]. Few studies examine the optimal time to initiate systemic therapy in children with uveitis.

Our primary objective is to describe timing of treatment using MTX and TNFi in children with CAU, and to examine potential factors associated with the addition of TNFi. Understanding the timing of TNFi and factors contributing to their initiation will inform decision-making related to children with uveitis and may improve visual outcomes.

\section{Materials and methods}

This retrospective study was conducted in a cohort of children with CAU that was either idiopathic or associated with JIA who were being followed prospectively in a larger uveitis epidemiology study. Approval was obtained by the Emory University Institutional Review Board (\#00017214) and conformed to the US Health Insurance Portability and Privacy Act requirements. Informed consent/assent was obtained from parents and children as appropriate.

\section{Subjects}

Children with uveitis were invited to participate during their pediatric rheumatology clinic visits at Emory Children's Center from September 2011 to July 2016. They were enrolled at varied time points after their uveitis diagnosis and were followed prospectively from time of enrollment during their usual follow-up appointments. For this study, inclusion criteria included: (1) a diagnosis of CAU that was either idiopathic (not associated with any systemic diseases (iCAU)) or associated with JIA (JIA-U), and (2) MTX treatment for uveitis at any point during the uveitis course. Exclusion criteria included: (1) refusal to participate, (2) MTX treatment for arthritis alone, (3) unknown reason for biologic treatment, and (4) acute anterior uveitis.

\section{Data collection}

Rheumatology and ophthalmology medical records were reviewed from time of diagnosis to enrollment, and every 3-6 months during follow-up. Data collected at baseline visit included date of birth, gender, self-reported race and ethnicity, JIA category by the International League of
Associations for Rheumatology (ILAR) classification in children with arthritis (oligoarticular, polyarticular, enthesitis-related, psoriatic, systemic, and undifferentiated) [28], uveitis characteristics (onset date, diagnosis date, laterality, location, ocular complications (i.e. cataracts, glaucoma, synechiae, band keratopathy, ocular hypertension, cystoid macular edema, and amblyopia), ocular surgeries, ocular exams (best-corrected visual acuity (BCVA), intraocular pressure, and anterior chamber (AC) cells score), and labs (antinuclear antibody (ANA), erythrocyte sedimentation rate (ESR)). Past and current use of topical and systemic medications were reviewed (name, dose, route of administration, start date, and discontinuation date).

We used Standardization of Uveitis Nomenclature criteria to define chronic anterior disease [29]. Anterior uveitis was defined as primary inflammation in the AC. Chronic uveitis was characterized by persistent uveitis with relapse $<3$ months after discontinuation of therapy. Active uveitis was defined as AC inflammation grade $>0.5+$ cells or $1-5$ cells in a field size of $1 \mathrm{~mm}$ by $1 \mathrm{~mm}$ slit beam. Inactive uveitis was defined as grade 0 cells or $<1$ cell in field. Primary outcome was defined as the addition of a TNFi to MTX for (1) persistent AC cells $>0.5+$ for $>3$ months while on MTX, (2) need for $>2$ drops a day of prednisolone acetate or other steroid equivalent for $>1$ month to maintain inactive uveitis, and/or (3) need for oral prednisone for $>$ 1 month to maintain inactive uveitis. BCVA was considered normal if $20 / 40$ or better, impaired if $20 / 50$ to better than $20 / 200$, severely impaired if worse than $20 / 200$, and blind if $20 / 400$ or worse.

Length of uveitis refers to time from uveitis diagnosis to last study visit. As routine ophthalmology screening is not performed in those without JIA, the length of uveitis may not be accurate in children without JIA. Follow-up data were collected at 3-6 months intervals (time of last study visit to current study visit) and during uveitis flares. Schedule of ophthalmology exams varied since visit intervals depended on uveitis activity and physician preference. We had limited data on the initial ocular exams since 21 patients had records available prior to enrollment.

\section{Statistical analysis}

Statistical analyses were conducted using SAS v. 9.4 for Windows (Cary, NC). Statistical significance was assessed at the 0.05 level unless otherwise noted and all tests were two-sided. Data are summarized using means and standard deviations, or counts and percentages, when appropriate. Data were assessed for normality using histograms and normal probability plots. Non-normally distributed data were presented as medians with associated 25 th and 75 th percentiles. Duration of medication treatment was calculated as time from start of medication to discontinuation or 
Table 1 Characteristics of children with JIA-associated uveitis and idiopathic chronic anterior uveitis on methotrexate with and without biologic therapy

\begin{tabular}{|c|c|c|c|}
\hline Characteristics & Overall & Methotrexate alone & $\begin{array}{l}\text { Methotrexate with } \\
\text { biologic }\end{array}$ \\
\hline$N(\%)$ unless otherwise specified & $N=46$ & $N=19(41 \%)$ & $N=27(59 \%)$ \\
\hline \multicolumn{4}{|l|}{ Demographics } \\
\hline Age at last follow-up, & 10.7 & 11.3 & 10.6 \\
\hline Median (25th-75th) & $(9.4-15.1)$ & $(9.4-16.3)$ & $(8.4-14.4)$ \\
\hline Female & $34(73.9 \%)$ & $13(68.4 \%)$ & $21(77.8 \%)$ \\
\hline \multicolumn{4}{|l|}{ Race } \\
\hline White & $29(63.0 \%)$ & $13(68.4 \%)$ & $16(59.3 \%)$ \\
\hline African American & $12(26.1 \%)$ & $3(15.8 \%)$ & $9(33.3 \%)$ \\
\hline Other & $5(10.9 \%)$ & $3(15.8 \%)$ & $2(7.4 \%)$ \\
\hline Hispanic & $8(17.4 \%)$ & $2(10.5 \%)$ & $6(22.2 \%)$ \\
\hline \multicolumn{4}{|l|}{ Year MTX started } \\
\hline Before 2013 & $25(54.4 \%)$ & $10(52.6 \%)$ & $15(55.6 \%)$ \\
\hline 2013 and beyond & $21(45.7 \%)$ & $9(47.4 \%)$ & $12(44.4 \%)$ \\
\hline \multicolumn{4}{|l|}{ Disease characteristics } \\
\hline \multicolumn{4}{|l|}{ Uveitis diagnosis } \\
\hline JIA-associated uveitis & $36(78.3 \%)$ & $16(84.2 \%)$ & $20(74.1 \%)$ \\
\hline Idiopathic chronic anterior uveitis & $10(21.7 \%)$ & $3(15.8 \%)$ & $7(25.9 \%)$ \\
\hline $\begin{array}{l}\text { Age at uveitis diagnosis (years), median } \\
\text { (25th-75th) }\end{array}$ & $4.9(3.7-7.4)$ & $6.2(4.5-10.4)$ & $4.4(3.4-6.7)$ \\
\hline Bilateral disease & $36(78.3 \%)$ & $14(73.7 \%)$ & $22(81.5 \%)$ \\
\hline \multicolumn{4}{|l|}{ Worst ocular exam } \\
\hline \multicolumn{4}{|l|}{ Anterior chamber cells $(n=45)$} \\
\hline $0 \& 0.5+$ & $6(13.3 \%)$ & $2(11.1 \%)$ & $4(14.8 \%)$ \\
\hline $1+$ and worse & $39(86.7 \%)$ & $16(88.9 \%)$ & $23(85.2 \%)$ \\
\hline Visual acuity $20 / 50$ or worse & $14(30.4 \%)$ & $6(31.6 \%)$ & $8(29.6 \%)$ \\
\hline Visual acuity $20 / 200$ or worse & $11(23.9 \%)$ & $2(10.5 \%)$ & $9(33.3 \%)$ \\
\hline Ocular complications, ever & $33(71.7 \%)$ & $13(68.4 \%)$ & $20(84.1 \%)$ \\
\hline Cataracts & $20(43.5 \%)$ & $8(42.1 \%)$ & $12(44.4 \%)$ \\
\hline Glaucoma or ocular hypertension & $13(28.3 \%)$ & $8(42.1 \%)$ & $5(18.5 \%)$ \\
\hline Synechiae & $24(54.2 \%)$ & $9(47.3 \%)$ & $15(55.6 \%)$ \\
\hline Band keratopathy & $18(39.1 \%)$ & $5(26.3 \%)$ & $13(48.2 \%)$ \\
\hline Cystoid macular edema & $10(21.7 \%)$ & $3(15.7 \%)$ & $7(25.9 \%)$ \\
\hline Other $^{1}$ & $11(23.9 \%)$ & $4(21.1 \%)$ & $7(25.9 \%)$ \\
\hline \multicolumn{4}{|l|}{ Labs } \\
\hline ANA positive $(n=45)$ & $21(46.7 \%)$ & $7(38.9 \%)$ & $14(51.9 \%)$ \\
\hline Earliest ESR $(n=45)$ & 14 & 20 & 14 \\
\hline Median (25th-75th) & $(7-32)$ & $(8-40)$ & $(4-25)$ \\
\hline \multicolumn{4}{|l|}{ Medication use, ever } \\
\hline Topical steroids & $45(97.8 \%)$ & $18(94.7 \%)$ & $27(100.0 \%)$ \\
\hline Dilating drops & $21(45.7 \%)$ & $8(42.1 \%)$ & $13(48.2 \%)$ \\
\hline Pressure drops & $7(15.2 \%)$ & $4(21.5 \%)$ & $3(11.1 \%)$ \\
\hline Oral steroids & $1(2.2 \%)$ & $0(0 \%)$ & $1(3.7 \%)$ \\
\hline Methotrexate, oral & $36(78.3 \%)$ & $13(68.4 \%)$ & $23(85.2 \%)$ \\
\hline Methotrexate, subcutaneous & $41(89.1 \%)$ & $17(89.5 \%)$ & $24(88.9 \%)$ \\
\hline Mycophenolate & $5(10.9 \%)$ & $2(10.5 \%)$ & $3(11.1 \%)$ \\
\hline Infliximab & $19(41.3 \%)$ & - & - \\
\hline
\end{tabular}


Table 1 (continued)

\begin{tabular}{llll}
\hline Characteristics & Overall & Methotrexate alone & $\begin{array}{l}\text { Methotrexate with } \\
\text { biologic }\end{array}$ \\
$N(\%)$ unless otherwise specified & $N=46$ & $N=19(41 \%)$ & $N=27(59 \%)$ \\
\hline Adalimumab & $11(23.9 \%)$ & - & - \\
Abatacept infusions & $2(4.4 \%)$ & - & - \\
Tocilizumab & $1(2.2 \%)$ & - & - \\
\hline
\end{tabular}

${ }^{1}$ Other includes: ambylopia, choriorental scaring, bullous retinoschisis, aphakia, and keratic precipitates

last study visit. Because requiring a biologic is a timedependent outcome, time to adding TNFi was described using estimates of survival derived from Kaplan-Meier survival curves with associated $95 \%$ confidence intervals. Cox proportional hazard $(\mathrm{PH})$ regression was used to identify risk factors associated with adding TNFi. The PH assumption was verified visually by examining the hazard plots and statistically by including the interaction between the logarithm of time and the covariate of interest in all univariate models. The $\mathrm{PH}$ assumption was considered suspect if the interaction between time and the covariate of interest was significant at the 0.1 level. If the $\mathrm{PH}$ assumption appeared to be violated, hazard ratios were estimated at specific time intervals (e.g. 3, 6, 12, 24, and 36 months).

\section{Results}

There were 51 children (41 (80\%) JIA-U and $10(20 \%)$ iCAU) in our cohort treated with MTX for uveitis alone or for both uveitis and arthritis. Five patients were excluded because their reason for discontinuing MTX or adding a TNFi was unrelated to uveitis or could not be verified. For subsequent analyses, we included 46 children with CAU (36 JIA-U and 10 iCAU) who started MTX for uveitis, and had complete ocular exam data prior to addition of TNFi.

\section{Characteristics of children treated with MTX}

Of the 46 children treated with MTX, 36 (78\%) had JIA-U (Table 1). JIA categories included 24 (67\%) oligoarticular persistent, 5 (14\%) enthesitis-related arthritis, 4 (11\%) polyarticular RF negative, and $3(8 \%)$ oligoarticular extended JIA. Ocular complications occurred in 33 (72\%) children during their disease course; vision loss, or a VA of $20 / 50$ and worse, (worst vision recorded in the more affected eye) occurred in 25 (56\%) children either secondary to inflammation or permanent ocular damage. Median duration of uveitis at last follow-up was 4.4 years (25th-75th: 2.7-7.6 years). Median duration of study follow-up was 2.4 years (25th-75th: 1.1-3.2 years).

\section{Medication use}

Topical glucocorticoids $(45,98 \%)$ were commonly prescribed; only one child (2\%) was treated with systemic glucocorticoids (Table 1). MTX was started a median of 5.0 months (range: 1.4-18.6) from uveitis diagnosis and was given for a median of 2.0 years (0.6-4.1) at last follow-up. Dose was $>0.5 \mathrm{mg} / \mathrm{kg} / \mathrm{dose}$ (maximum $30 \mathrm{mg}$ ) in $91 \%$ of children, and administered most frequently by subcutaneous route $(41,89 \%)$. Mycophenolate was the only other disease modifying anti-rheumatic drug prescribed $(5,11 \%)$.

\section{Biologic use}

At last follow-up, $19(41 \%)$ were treated with MTX exclusively, and 27 (59\%) were escalated to TNFi for (1) persistent AC cells $>0.5+$ for $>3$ months while on MTX, (2) need for $>2$ drops a day of prednisolone acetate or other topical steroid equivalent for $>1$ month to maintain inactive uveitis, and/or (3) need for oral prednisone for $>$ 1 month to maintain inactive uveitis. TNFi was the initial biologic in all children. The first TNFi was started a median of 3.6 years (range: 0.3-13.0) from diagnosis and was given for a median of 1.6 years (range: 0.03-7.8) based on last follow-up. Four patients ( $9 \%$ of total cohort, $15 \%$ of those requiring $\mathrm{TNFi}$ ) required a second $\mathrm{TNFi}$. Other biologics prescribed included abatacept (2, 4\%) and tocilizumab $(1,2 \%)$.

Infliximab was the most common $(19,41 \%)$ initial biologic. It was started at a median of 2.4 years (range: 0.4 8.5) from uveitis diagnosis and given for a median of 1.7 years (range: $0.1-7.8$ ). Dose was $10 \mathrm{mg} / \mathrm{kg} / \mathrm{dose}$ in seven children, $7.5 \mathrm{mg} / \mathrm{kg} / \mathrm{dose}$ in three, and $5 \mathrm{mg} / \mathrm{kg} / \mathrm{dose}$ in eight; all patients received Infliximab every 4 weeks.

Adalimumab was the second most common initial biologic $(11,24 \%)$. It was started a median of 3.8 years (range: 1.2-13.0) from uveitis diagnosis and escalated to weekly injections from every other week in three of eight children. Three children were still on concomitant MTX and one on mycophenolate at last follow-up. 
Table 2 Timing of methotrexate and TNFi use in children with JIA-associated uveitis and idiopathic chronic anterior uveitis $(N=46)$

\begin{tabular}{lll}
\hline Drug & $N(\%)$ or median $(25$ th-75th) & Cumulative frequency \\
\hline MTX & & \\
Time from diagnosis, months & & - \\
Median (25th-75th) & $2(4.4 \%)$ & $2(4.4 \%)$ \\
At diagnosis & $24(52.2 \%)$ & $26(56.5 \%)$ \\
Within 6 months of diagnosis & $6(13.0 \%)$ & $32(69.6 \%)$ \\
Within 1 year of diagnosis & $9(19.6 \%)$ & $41(89.1 \%)$ \\
$1-<3$ years after diagnosis & $4(8.7 \%)$ & $45(97.8 \%)$ \\
$3-5$ years after diagnosis & $1(2.2 \%)$ & $46(100 \%)$ \\
$>5$ years after diagnosis & & - \\
TNFi (n = 27) & 42.8 & \\
Time from diagnosis, months & $(13.9-69.5)$ & $3(11.1 \%)$ \\
Median (25th-75th) & $3(11.1 \%)$ & $6(22.2 \%)$ \\
Within 6 months of diagnosis & $3(11.1 \%)$ & $13(48.2 \%)$ \\
Within 1 year of diagnosis & $7(25.9 \%)$ & $20(74.1 \%)$ \\
$1-<3$ years after diagnosis & $7(25.9 \%)$ & $27(100.0 \%)$ \\
$3-5$ years after diagnosis & $7(25.9 \%)$ & - \\
$>5$ years after diagnosis & 19.3 & $23(85.2 \%)$ \\
Time from MTX use, & $(7.1-46.9)$ & $5(18.5 \%)$ \\
Median (25th-75th) & $5(18.5 \%)$ & $9(33.3 \%)$ \\
Within 6 months of MTX & $4(14.8 \%)$ & $18(66.7 \%)$ \\
Within 1 year of MTX & $9(33.3 \%)$ & \\
$1-<3$ years after MTX & $5(18.5 \%)$ & \\
3-5 years after MTX & $4(14.8 \%)$ & \\
$>5$ years after MTX & & \\
\hline
\end{tabular}

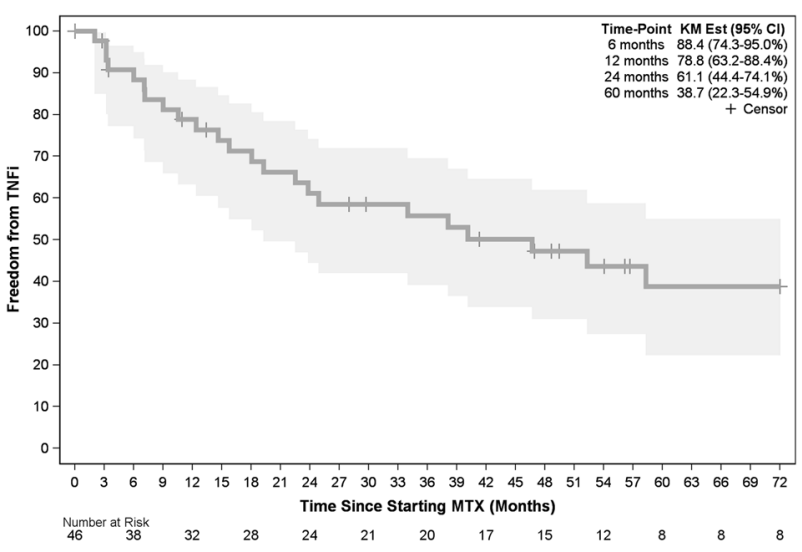

Fig. 1 Kaplan-Meier curve showing freedom from TNFi and number at risk. Gray bands represent the $95 \%$ confidence limits

\section{Survival analysis}

MTX was initiated at a median of 5.0 months (25th-75th percentile: 1.4-18.6 months) following diagnosis (Table 2). Cumulatively, MTX was started in $57 \%$ of children within 6 months of uveitis diagnosis, $70 \%$ within 1 year, and $89 \%$ within $1-<3$ years.
Twenty-seven patients $(56 \%)$ required addition of TNFi at a median of 19 months (25th-75th percentile: 7.1-46.9) following MTX. Kaplan-Meier estimates suggest that cumulatively, $12 \%$ added TNFi within 6 months of MTX, $21 \%$ within 1 year, $39 \%$ within 2 years, and $61 \%$ within 5 years (Fig. 1).

Because duration of follow-up differed, we explored the relationship between potential risk factors and addition of TNFi using Cox PH regression models. For some variables, the PH assumption was violated (i.e., the hazard varied over follow-up) and, as a result, hazard ratios are presented at different points during follow-up. Children with iCAU were more likely to initiate TNFi early in their MTX course compared with JIA-U; however, later in follow-up, the risk of requiring a TNFi was similar between the two groups (Fig. 2a). Specifically, at 3 months, the hazard of requiring a TNFi after initiation of MTX was six times higher in iCAU (hazard's ratio (HR) 6.06; 95\% confidence interval (CI) (1.25-29.41)), but by 12 months the risk was no longer significant (HR 2.16; 95\% CI (0.84-5.56)) (Table 3). Two years after initiating MTX, the hazard of requiring a TNFi was similar in both groups ( $\mathrm{HR}=1.28$; $95 \%$ CI $(0.44$ $3.75)$ ). Using median age of diagnosis of the entire cohort 
Fig. 2 a Kaplan-Meier curves showing freedom from $\mathrm{TNFi}$ stratified by diagnosis group. Black line represents patients with JIA-U and gray line represents patients with idiopathic CAU. b KaplanMeier curve showing freedom from TNFi stratified by gender. Black line represents males and gray line represents females. In both panels, shaded areas represent the $95 \%$ confidence intervals
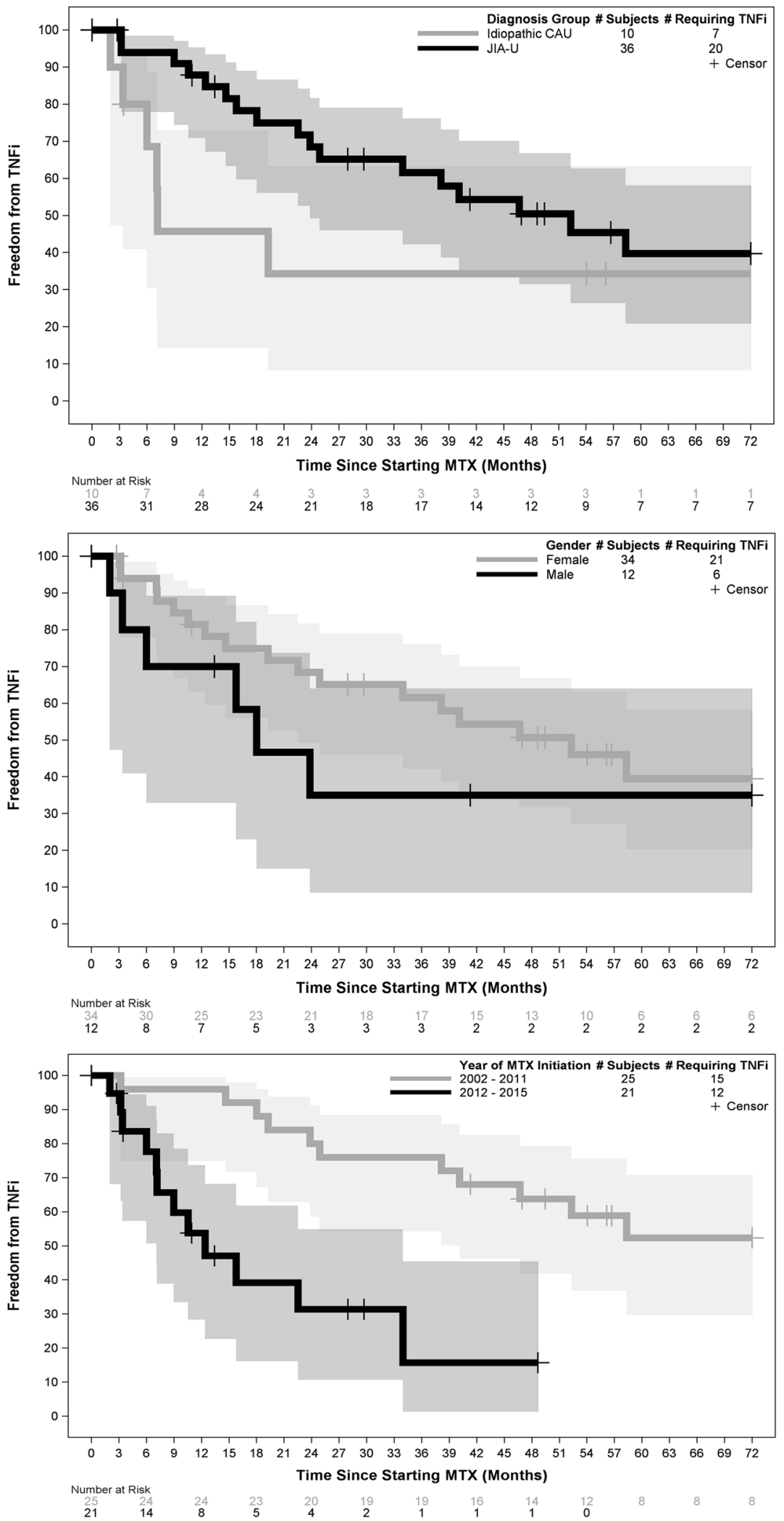

(4.9 years), age at diagnosis $(<5$ years vs. $\geq 5)$ was not associated with TNFi addition. However, variable duration of follow-up may have affected results. Females appeared less likely to require TNFi early in their disease compared with males, but this was not significant over time (Fig. 2b). Additional analysis showed that race, ethnicity, bilateral 
Table 3 Analysis of time to initiation of TNFi using Cox proportional hazard regression
Characteristics $N=$ unless otherwise specified $N(\%)$ unless otherwise specified

\begin{tabular}{|c|c|c|c|}
\hline \multicolumn{4}{|l|}{ Demographics } \\
\hline \multicolumn{4}{|l|}{ Gender, female } \\
\hline 3 Months & 0.21 & $(0.04-1.03)$ & 0.054 \\
\hline 6 Months & 0.4 & $(0.13-1.28)$ & 0.124 \\
\hline 12 Months & 0.77 & $(0.28-2.10)$ & 0.609 \\
\hline 24 Months & 1.48 & $(0.43-5.10)$ & 0.538 \\
\hline 36 Months & 2.15 & $(0.48-9.52)$ & 0.316 \\
\hline \multicolumn{4}{|l|}{ Race } \\
\hline White (reference) & - & - & - \\
\hline African American & 1.68 & $(0.73-3.86)$ & 0.219 \\
\hline Other & 0.44 & $(0.10-1.93)$ & 0.278 \\
\hline Hispanic & 1.54 & $(0.61-3.86)$ & 0.357 \\
\hline \multicolumn{4}{|l|}{ Treatment Era } \\
\hline 2002-2011 (reference) & - & - & - \\
\hline 2012-2015 & 4.99 & $(1.97-12.67)$ & $<0.001$ \\
\hline \multicolumn{4}{|l|}{ Disease characteristics } \\
\hline \multicolumn{4}{|l|}{ Type of uveitis } \\
\hline Idiopathic chronic anterior uveitis vs. JIA-associated uveitis & & & -- \\
\hline 3 Months & 6.06 & $(1.25-29.41)$ & 0.025 \\
\hline 6 Months & 3.62 & $(1.13-11.55)$ & 0.03 \\
\hline 12 Months & 2.16 & $(0.84-5.57)$ & 0.111 \\
\hline 24 Months & 1.28 & $(0.44-3.75)$ & 0.653 \\
\hline 36 Months & 0.95 & $(0.27-3.39)$ & 0.936 \\
\hline Age at uveitis diagnosis (years), per 1 year increase & 1 & $(0.89-1.13)$ & 0.96 \\
\hline Age $<5$ at diagnosis & 1.31 & $(0.55-3.14)$ & 0.547 \\
\hline Bilateral disease & 0.84 & $(0.31-2.26)$ & 0.723 \\
\hline \multicolumn{4}{|l|}{ Initial ocular exam (within 2 months of $\mathrm{dx}$ ) } \\
\hline$\geq 1+$ cells at first exam $(n=17)$ & 3.76 & $(0.44-32.57)$ & \\
\hline Complications at initial exam, $N(\%)(n=21)$ & 2.77 & $(0.74-10.33)$ & 0.129 \\
\hline \multicolumn{4}{|l|}{ Labs } \\
\hline ANA positive $(n=45)$ & 1.07 & $(0.50-2.29)$ & 0.872 \\
\hline Earliest ESR per 1 unit & 0.99 & $(0.97-1.01)$ & 0.368 \\
\hline
\end{tabular}

disease, and labs were not associated with time to addition of TNFi.

To account for changes in prescribing practice over time, we explored time to initiation of TNFi and divided our cohort into roughly two equally sized cohorts based on the year they started MTX (2002-2011 and 2012-2015). Results from survival analysis showed that median time to TNFi use in the earlier cohort was 74.8 months $(95 \% \mathrm{CI}$ : 40.1-136.6) and was significantly longer than the more recent era (median time to TNFi: 12.4 ; 95\% CI: 7.1-34.0; $p<0.001)$. Although there was no difference in the proportion of patients receiving a TNFi in the two era cohorts (60 vs. 57\%), Fig. 2c shows that TNFi is initiated much sooner in the more recent era cohort (HR: 4.99; $95 \%$ CI: $1.97-12.67 ; p<0.001)$.

\section{Treatment-related factors}

In iCAU, median time from diagnosis to first rheumatology visit was 0.48 years (5-6 months), IQR (2 months-1.5 years), thus $25 \%$ are evaluated by rheumatology within 2 months of diagnosis. One patient was started on MTX by their ophthalmologist prior to seeing a rheumatologist. In the remaining nine patients, MTX was started in 44\% (4/9) within 2 weeks of first rheumatology visit, and $67 \%(6 / 9)$ within 2 months. However, there was no association between time from uveitis diagnosis to MTX initiation and addition of TNFi $(\mathrm{HR}=1.02 ; 95 \%$ CI $(0.79-1.31)$ $p=0.909)$.

In JIA-U, 16 (44\%) were managed by a rheumatologist for arthritis prior to their uveitis diagnosis. Of those, $69 \%$ 
started MTX within 6 months of uveitis diagnosis. For the 20 children not seen by a rheumatologist prior to their uveitis diagnosis $(56 \%), 13(65 \%)$ had their first visit at our institution. Median time from uveitis diagnosis to first rheumatology visit was 0.48 years ( 6 months), (range 1 week to 1.7 years). Twenty-three percent (3/13) started MTX within 2 months of their first visit, which is similar to children with iCAU.

\section{Discussion}

We report on the timing of MTX initiation and TNFi addition in a cohort of children with CAU, wherein MTX was started a median of 5 months from uveitis diagnosis. TNFi was added in 59\% of children on MTX and initiated at a median of 19 months from MTX start. Children with iCAU and males required TNFi addition earlier in their uveitis course. MTX is known to be effective for uveitis, but the optimal timing to add TNFi therapy and factors associated with the need for TNFi require further investigation.

Ideal timing of systemic treatment in uveitis is unknown. Ocular complications and vision loss remain a potential consequence of pediatric uveitis, despite the availability and established benefits of effective treatment. In our cohort, $70 \%$ were on MTX and $22 \%$ on TNFi within the first year of uveitis diagnosis. Ocular complications $(70 \%)$ and vision loss $(30 \%)$ were present at some point during the uveitis course, which is similar to previous reports $[4-8,30]$. In recent-onset JIA, early aggressive treatment led to inactive disease and clinical remission on medication [31, 32]. In uveitis, reports show that immunomodulatory therapy (IMT) improves visual outcomes. Gregory et al. demonstrated that immunosuppressive drugs decreased risk for vision loss, or a VA of $\leq 20 / 50$ (HR, 0.40 ; 95\% CI, $0.21-$ $0.75, p<0.01)$ [6]. Thorne et al. [1] reported that IMT reduced the risk for complications and blindness in the better eye (RR, $0.41 ; 95 \%$ CI $0.11-1.15, p=0.07)$. Active inflammation at follow-up increased risk for visual impairment and blindness. Ramanan et al. demonstrated that adding adalimumab to MTX delayed time to treatment failure, that treatment failure was lower in children on both adalimumab and MTX, and that there was a significant reduction in topical glucocorticoids compared with children on MTX alone [16]. These studies support the use of systemic treatment for early disease control. Further study on the optimal timing of treatment to prevent ocular complications and vision loss should be considered.

TNFi is commonly added in children with severe uveitis or who fail or are intolerant of MTX. Risk factors for a severe uveitis course and poor prognosis in JIA-U include young age at onset, male gender, short interval between arthritis and uveitis, ANA positivity, severe uveitis at presentation, non-oligoarticular JIA, delay in presentation to a subspecialist, and African American race [1, 6, 7, 30, 3337]. Data are sparse on factors associated with MTX failure. In our cohort, children with iCAU required TNFi earlier compared with those with JIA. Children with iCAU, however, often present with increased ocular sequelae as children with JIA undergo regular ophthalmology screening, leading to earlier detection of uveitis. Interestingly, those who did not require TNFi by 18 months were unlikely to do so, although this may be affected by follow-up duration. Studies on relapse and remission after medication discontinuation show that children with JIA-U are less likely to sustain remission [38]. There was a trend for males to add TNFi earlier than females. Although uveitis is less common in males, they may experience more severe disease $[7,8,39,40]$. Further exploration is needed to define the population of children with greatest need for early TNFi.

Factors such as time to referral to a uveitis specialist and/ or rheumatologist, insurance difficulties, patient/parent preference, or MTX intolerance/side effects can influence initiation of TNFi. In our cohort of children seen by an ophthalmologist before a rheumatologist, referral to rheumatology occurred within 5-6 months of diagnosis and treatment initiated after a median of 2 months from first evaluation, which differs from children under the care of a rheumatologist at uveitis diagnosis. In 21 children for whom we had records of the initial ocular exam, $12(57 \%)$ had ocular complications at presentation, and $\sim 50 \%(10 / 21)$ had keratic precipitates (data not shown). The addition of TNFi was also affected by medication accessibility, as there was no FDA approved biologic for pediatric uveitis at time of this study. Adalimumab was approved for adults with intermediate, posterior, and panuveitic non-infectious uveitis in 2016, but there is no pediatric uveitis indication until recently [41, 42]. To date, there is only one RCT of MTX and adalimumab use in children with uveitis, which showed the benefit of combination therapy with both agents compared with MTX alone [16]. Delayed referral to subspecialists and availability of drug can impact initiation of treatment.

Collaboration and communication between rheumatologists and ophthalmologists remains crucial. The American Academy of Pediatrics recommends routine ophthalmology screening for uveitis in children with JIA [43]. However, in those without JIA, regular eye screening is not routinely performed. In our cohort of iCAU, the median time to the first rheumatology visit was 5-6 months after diagnosis, and $75 \%$ started MTX within 2 months of their visit. Although there was no association between time from uveitis diagnosis to initiation of MTX and risk of addition of TNFi $(\mathrm{HR}=1.02 ; 95 \% \mathrm{CI}(0.79-1.31) p=0.909)$, most children with iCAU required TNFi earlier in their disease. This may be owing to severe disease at presentation secondary to 
underlying pathogenesis, delayed ophthalmology screening, or delayed rheumatology referral.

We acknowledge that the need to initiate biologics may not depend solely on uveitis severity. Other factors to consider include glucocorticoid responsiveness, tolerance or compliance with non-biologic therapy, physician preference, and clinical support to initiate and monitor treatment. In addition, JIA studies report that pharmacogenetics contributes to the variability in clinical response to MTX $[44,45]$. MTX is effective in $\sim 73 \%$ of children with autoimmune chronic uveitis [17]. Genetic variation may account for the variability in drug response and toxicity, thus, underlying ontology and the need for a personalized medicine approach may have an important role in treatment.

Our study had several limitations. Recent data have shown the benefit of combination therapy using both MTX and adalimumab in the treatment of children with uveitis. Given the rapidly changing landscape in the management of pediatric uveitis, increased availability of biologics for treatment, and growing evidence that timely treatment improves outcomes, our practice has evolved to initiate biologic therapy sooner, especially in those with severe disease at presentation. Thus, our study may not reflect recent treatment patterns. As a tertiary center, there is a potential selection bias for children with severe disease, and a referral bias owing to our specialized care in uveitis. There is close collaboration between rheumatologists and ophthalmologists at our center, with shared decision-making for the initiation of TNFi, and monitoring of uveitis activity and immunosuppressive therapy. This may not reflect the experience of all centers in the use of systemic treatment. Referral times may also be decreased compared with other centers. Enrollment at variable time points, as well as initial evaluations at outside centers, contributed to missing medical records from the first ophthalmology visit for some patients. Thus, we are unable to ascertain in all children whether complications and vision loss were present at the initial exam, outside of our baseline examination at our institution or occurred despite treatment. The availability of biologic therapy and the evidence of the benefit of initiating TNFi changed through the course of the study. This is evident in our findings of median time to TNFi use in children prior to 2011 was 74 months compared with only 12 months in children treated in 2011 and later.

Despite these limitations, one strength of this study is that only children diagnosed with uveitis after 1998, when TNFi was available, were included. Our cohort also included both children with iCAU or JIA-U. In addition, we followed our patients prospectively after enrollment.

We report on medication use in children with CAU and describe time to initiation of MTX and TNFi. Specific populations may benefit from early treatment. Thus, timely referral to subspecialists, close collaboration between rheumatologists and ophthalmologists, and a lower threshold to initiating systemic therapy, especially in those at increased risk for severe disease, is crucial. Studies on factors that influence the need for additional therapy and on the optimal time to initiate treatment should be considered. Timely control of inflammation may improve visual outcomes by preventing tissue damage, subsequent ocular complications, and loss of vision.

\section{Summary}

What was known before:

- Little is known about the optimal time to initiate systemic therapy in children with uveitis.

- Many children fail methotrexate and require the addition of anti-tumor necrosis factor alpha inhibitors to treat their uveitis.

- Few studies describe risk factors associated with the need to add anti-tumor necrosis factor alpha inhibitors.

\section{What this study adds:}

- This study describes the natural history of methotrexate and anti-tumor necrosis factor alpha inhibitor medications use in a cohort of children with chronic anterior non-infectious uveitis, the most common type of pediatric uveitis.

- This study explores potential factors that increase risk of methotrexate failure and need for biologic therapy.

Acknowledgements We thank Anna Tramposch and Bessie Frias for their assistance with data collection and data entry.

Funding Dr. Angeles-Han is supported by Award Number K23EY021760 from the National Eye Institute, the Rheumatology Research Foundation, and the CCHMC Research Innovation and Pilot Fund. Dr. Prahalad is supported by The Marcus Foundation Inc. Atlanta GA.

\section{Compliance with ethical standards}

Conflict of interest Dr. Steven Yeh is a consultant for Santen, Inc. and Clearside Biomedical. Dr. Sampath Prahalad is on an advisory committee for Novartis.

\section{References}

1. Thorne JE, Woreta F, Kedhar SR, Dunn JP, Jabs DA. Juvenile idiopathic arthritis-associated uveitis: incidence of ocular complications and visual acuity loss. Am J Ophthalmol. 2007;143:840-6. 
2. Woreta F, Thorne JE, Jabs DA, Kedhar SR, Dunn JP. Risk factors for ocular complications and poor visual acuity at presentation among patients with uveitis associated with juvenile idiopathic arthritis. Am J Ophthalmol. 2007;143:647-55.

3. Thorne JE, Suhler E, Skup M, Tari S, Macaulay D, Chao J, et al. Prevalence of noninfectious uveitis in the United States. JAMA Ophthalmol. 2016;21287:1237-45.

4. Angeles-Han ST, McCracken C, Yeh S, Jenkins K, Stryker D, Rouster-Stevens K, et al. Characteristics of a cohort of children with juvenile idiopathic arthritis and JIA-associated uveitis. Pediatr Rheumatol Online J. 2015;13:1-10.

5. Angeles-Han ST, Pelajo CF, Vogler LB, Rouster-Stevens K, Kennedy C, Ponder L, et al. Risk markers of juvenile idiopathic arthritis-associated uveitis in the Childhood Arthritis and Rheumatology Research Alliance (CARRA) registry. J Rheumatol. 2013;40:2088-96.

6. Gregory 2ndAC, Kempen JH, Daniel E, Kacmaz RO, Foster CS, Jabs DA, et al. Risk factors for loss of visual acuity among patients with uveitis associated with juvenile idiopathic arthritis: the Systemic Immunosuppressive Therapy for Eye Diseases Study. Ophthalmology. 2013;120:186-92.

7. Edelsten C, Lee V, Bentley CR, Kanski JJ, Graham EM. An evaluation of baseline risk factors predicting severity in juvenile idiopathic arthritis associated uveitis and other chronic anterior uveitis in early childhood. Br J Ophthalmol. 2002;86:51-6.

8. Holland GN, Denove CS, Yu F. Chronic anterior uveitis in children: clinical characteristics and complications. Am J Ophthalmol. 2009;147:667-78 e5.

9. Bou R, Adan A, Borras F, Bravo B, Calvo I, De Inocencio J, et al. Clinical management algorithm of uveitis associated with juvenile idiopathic arthritis: interdisciplinary panel consensus. Rheumatol Int. 2015;35:777-85.

10. Heiligenhaus A, Michels H, Schumacher C, Kopp I, Neudorf U, Niehues $\mathrm{T}$, et al. Evidence-based, interdisciplinary guidelines for anti-inflammatory treatment of uveitis associated with juvenile idiopathic arthritis. Rheumatol Int. 2012;32:1121-33.

11. Levy-Clarke G, Jabs DA, Read RW, Rosenbaum JT, Vitale A, Van Gelder RN. Expert panel recommendations for the use of anti-tumor necrosis factor biologic agents in patients with ocular inflammatory disorders. Ophthalmology. 2014;121:785-96 e3.

12. Henderson LA, Zurakowski D, Angeles-Han ST, Lasky A, Rabinovich CE, Lo MS. Medication use in juvenile uveitis patients enrolled in the Childhood Arthritis and Rheumatology Research Alliance Registry. Pediatr Rheumatol Online J. 2016; 14:9.

13. Weiss AH, Wallace CA, Sherry DD. Methotrexate for resistant chronic uveitis in children with juvenile rheumatoid arthritis. J Pediatr. 1998;133:266-8.

14. Heiligenhaus A, Mingels A, Heinz C, Ganser G. Methotrexate for uveitis associated with juvenile idiopathic arthritis: value and requirement for additional anti-inflammatory medication. Eur $\mathbf{J}$ Ophthalmol. 2007;17:743-8.

15. Foeldvari I, Wierk A. Methotrexate is an effective treatment for chronic uveitis associated with juvenile idiopathic arthritis. J Rheumatol. 2005;32:362-5.

16. Ramanan AV, Dick AD, Jones AP, McKay A, Williamson PR, Compeyrot-Lacassagne $\mathrm{S}$, et al. Adalimumab plus methotrexate for uveitis in juvenile idiopathic arthritis. $\mathrm{N}$ Engl $\mathrm{J}$ Med. 2017;376:1637-46.

17. Simonini G, Paudyal P, Jones GT, Cimaz R, Macfarlane GJ. Current evidence of methotrexate efficacy in childhood chronic uveitis: a systematic review and meta-analysis approach. Rheumatol. 2013;52:825-31.

18. Simonini G, Druce K, Cimaz R, Macfarlane GJ, Jones GT. Current evidence of anti-tumor necrosis factor alpha treatment efficacy in childhood chronic uveitis: a systematic review and meta- analysis approach of individual drugs. Arthritis Care Res. 2014;66:1073-84.

19. Zannin ME, Birolo C, Gerloni VM, Miserocchi E, Pontikaki I, Paroli MP, et al. Safety and efficacy of infliximab and adalimumab for refractory uveitis in juvenile idiopathic arthritis: 1-year followup data from the Italian Registry. J Rheumatol. 2013;40:74-9.

20. Rajaraman RT, Kimura Y, Li S, Haines K, Chu DS. Retrospective case review of pediatric patients with uveitis treated with infliximab. Ophthalmology. 2006;113:308-14.

21. Kahn P, Weiss M, Imundo LF, Levy DM. Favorable response to high-dose infliximab for refractory childhood uveitis. Ophthalmology. 2006;113:860-4 e2.

22. Ardoin SP, Kredich D, Rabinovich E, Schanberg LE, Jaffe GJ. Infliximab to treat chronic noninfectious uveitis in children: retrospective case series with long-term follow-up. Am J Ophthalmol. 2007;144:844-9.

23. Vazquez-Cobian LB, Flynn T, Lehman TJ. Adalimumab therapy for childhood uveitis. J Pediatr. 2006;149:572-5.

24. García-De-Vicuña C, Díaz-Llopis M, Salom D, Bou R, DíazCascajosa J, Cordero-Coma M, et al. Usefulness of adalimumab in the treatment of refractory uveitis associated with juvenile idiopathic arthritis. Mediators Inflamm. 2013;2013:560632.

25. Bravo-Ljubetic L, Peralta-Calvo J, Noval S, Pastora-Salvador N, Abelairas-Gomez J, Merino R. Adalimumab therapy for refractory childhood uveitis. J AAPOS. 2013;17:456-9.

26. Magli A, Forte R, Navarro P, Russo G, Orlando F, Latanza L, et al. Adalimumab for juvenile idiopathic arthritis-associated uveitis. Graefes Arch Clin Exp Ophthalmol. 2013;251:1601-6.

27. Tynjala P, Kotaniemi K, Lindahl P, Latva K, Aalto K, Honkanen $\mathrm{V}$, et al. Adalimumab in juvenile idiopathic arthritis-associated chronic anterior uveitis. Rheumatol. 2008;47:339-44.

28. Petty RE, Southwood TR, Manners P, Baum J, Glass DN, Goldenberg $\mathrm{J}$, et al. International League of Associations for Rheumatology classification of juvenile idiopathic arthritis: second revision, Edmonton, 2001. J Rheumatol. 2004;31:390-2.

29. Jabs DA Standardization of uveitis nomenclature for reporting clinical data. Results of the first international workshop. Am J Ophthalmol. 2005;140:509-16.

30. Vitale AT, Graham E, de Boer JH. Juvenile idiopathic arthritisassociated uveitis: clinical features and complications, risk factors for severe course, and visual outcome. Ocul Immunol Inflamm. 2013;21:478-85.

31. Wallace CA, Giannini EH, Spalding SJ, Hashkes PJ, O’Neil KM, Zeft AS, et al. Trial of early aggressive therapy in polyarticular juvenile idiopathic arthritis. Arthritis Rheum. 2012;64:2012-21.

32. Tynjälä $P$, Vähäsalo $P$, Tarkiainen $M$, Kröger L, Aalto K, Malin $\mathrm{M}$, et al. Aggressive combination drug therapy in very early polyarticular juvenile idiopathic arthritis (ACUTE-JIA): a multicentre randomised open-label clinical trial. Ann Rheum Dis. 2011;70:1605-12.

33. Dana MR, Merayo-Lloves J, Schaumberg DA, Foster CS. Visual outcomes prognosticators in juvenile rheumatoid arthritisassociated uveitis. Ophthalmology. 1997;104:236-44.

34. Heiligenhaus A, Niewerth M, Ganser G, Heinz C, Minden K, Ganser G, et al. Prevalence and complications of uveitis in juvenile idiopathic arthritis in a population-based nation-wide study in Germany: Suggested modification of the current screening guidelines. Rheumatology. 2007;46:1015-9.

35. Ayuso VK, Ten Cate HA, van der Does P, Rothova A, de Boer $\mathrm{JH}$. Male gender and poor visual outcome in uveitis associated with juvenile idiopathic arthritis. Am J Ophthalmol. 2010; 149:987-93.

36. Zannin ME, Buscain I, Vittadello F, Martini G, Alessio M, Orsoni JG, et al. Timing of uveitis onset in oligoarticular juvenile idiopathic arthritis (JIA) is the main predictor of severe course uveitis. Acta Ophthalmol. 2012;90:91-5. 
37. Angeles-Han ST, McCracken C, Yeh S, Jenkins K, Stryker D, Travers $\mathrm{C}$, et al. The association of race with childhood uveitis. Am J Ophthalmol. 2015;160:919-928.e1.

38. Simonini G, Bracaglia C, Cattalini M, Taddio A, Brambilla A, De Libero C, et al. Predictors of relapse after discontinuing systemic treatment in childhood autoimmune chronic uveitis. J Rheumatol. 2017;44:822-6.

39. Kalinina Ayuso V, ten Cate HAT, van der Does P, Rothova A, de Boer JH. Male gender as a risk factor for complications in uveitis associated with juvenile idiopathic arthritis. Am J Ophthalmol. 2010;149:994-.e5.

40. Zulian F, Martini G, Falcini F, Gerloni V, Zannin ME, Pinello L, et al. Early predictors of severe course of uveitis in oligoarticular juvenile idiopathic arthritis. J Rheumatol. 2002;29:2446-53.

41. Jaffe GJ, Dick AD, Brézin AP, Nguyen QD, Thorne JE, Kestelyn $\mathrm{P}$, et al. Adalimumab in patients with active noninfectious uveitis. N Engl J Med. 2016;375:932-43.
42. Nguyen QD, Merrill PT, Jaffe GJ, Dick AD, Kurup SK, Sheppard $\mathrm{J}$, et al. Adalimumab for prevention of uveitic flare in patients with inactive non-infectious uveitis controlled by corticosteroids (VISUAL II): a multicentre, double-masked, randomised, placebo-controlled phase 3 trial. Lancet. 2016; 388:1183-92.

43. Cassidy J, Kivlin J, Lindsley C, Nocton J. Ophthalmologic examinations in children with juvenile rheumatoid arthritis. Pediatrics. 2006;117:1843-5.

44. Cobb J, Cule E, Moncrieffe H, Hinks A, Ursu S, Patrick F, et al. Genome-wide data reveal novel genes for methotrexate response in a large cohort of juvenile idiopathic arthritis cases. Pharm J. 2014;14:356-64.

45. Becker ML, Leeder JS. Developmental pharmacogenetics in pediatric rheumatology: utilizing a new paradigm to effectively treat patients with juvenile idiopathic arthritis with methotrexate. Hum Genomics Proteom. 2010;2010:257120. 